\title{
Student attrition in gender atypical fields of study. A matter of lacking social integration or pressure from significant others?
}

Regina Jusri ${ }^{1}$

[currently under review]

Keywords: gender inequality, university student attrition, social integration, significant others

\begin{abstract}
In most European countries, the choice of college majors is highly segregated by gender. Only a small amount of students enrolls in a gender atypical major. Previous studies indicated that these students may have a higher attrition rate than their counterparts in gender typical majors. In this paper, I investigated whether this higher attrition rate is caused by lower levels of social integration and pressure from significant others. I built a synthesis of Tinto's model of student drop out and Kanter's theory of tokenism and drew on theories of comparative reference groups to test these hypotheses. Using data from the German National Educational Panel Study, which follows undergraduate students who enrolled in 2010, I conducted discrete time survival analysis. I found that men and women in gender atypical majors have a higher risk to switch to a more gender balanced major and - only for women - to drop out than students in gender typical majors. Low levels of social integration and disapproval of the major by significant others increase the attrition risk. However, these two aspects do not contribute to the explanation of the higher attrition risk of students in gender atypical majors. Alternative explanations are discussed.
\end{abstract}

\footnotetext{
${ }^{1}$ Leibniz-Institute for Educational Trajectories, Regina.Jusri@lifbi.de
} 


\section{Introduction}

In most European countries, women are overrepresented in higher education (OECD, 2018). However, the choice of college majors is still highly segregated by gender. Women are more likely to enter health studies, humanities, education and fine arts programs, while men are more often attracted to STEM-fields (science, technology, engineering and mathematics) (Bloom, 2013). These choices are consistent with gender stereotypes. Women are believed to have inferior mathematical or technological skills which make them less suited to STEM-fields. In contrast, gender beliefs suggest that men have lesser abilities in caregiving and communication and hence, are less suited for fields associated with those skills (Correll, 2001). Overall, men and women rarely choose a gender atypical field of study. Furthermore, some empirical studies suggests that these students show a higher non-completion risk than students in gender typical majors (Bloom, 2013; Meyer et al., 2019). However, results of previous studies are not consistent. Additionally, the causes for the sometimes found higher attrition risk have not yet been fully identified.

The objective of this article is to find out whether men and women in gender atypical majors experience a higher attrition risk than their peers in gender typical majors. Furthermore, I investigate whether social integration with fellow students and pressure from significant others contribute to the higher attrition risk of women and men in gender atypical majors using Germany as a case study. I will differentiate between distinct forms of non-completion, namely, the decision to drop out from higher education and the decision to switch majors.

Low social integration is one of the major determinants of student drop out from higher education (Tinto, 1975). To uncover whether differences in social integration contribute to the lower persistence rate of students in gender atypical majors, I will build a synthesis of Tinto's model of student drop out (1975) and Kanter's model of Tokenism (1977a). I will apply this theory to women and men in gender atypical majors and argue that their minority status leads to lower levels of social integration, and consequently higher drop out risk. Furthermore, significant others and their often traditional gender norms exert influence on students' choice of college majors (Gabay-Egozi et al., 2015). I will apply reference group theories to the context of non-completion decisions. I expect that students in gender atypical majors are exposed to pressure from significant others to leave their major because their major choice violates traditional gender norms. Additionally, I argue that majors with a more balanced 
gender composition, or gender typical majors, exert a pull-factor on students in gender atypical majors. I expect that these students - when switching majors and not dropping out completely - will switch to a major with a higher proportion of students of their gender. I will conduct within-gender comparisons to address these research questions.

My contribution to the existing literature is threefold. First, I will provide a synthesis of two widely applied theories, the theory of tokenism and Tinto's model of university drop out. I will test empirically whether such a synthesis sheds light on the mechanisms causing the high attrition risk of students in gender atypical majors. In contrast to previous studies, I will use a longitudinal data set with a continuous measurement of social integration, the student cohort of the German National Educational Panel Study ${ }^{1}$, to test these assumptions in depth. Second, I will investigate the impact of significant others on drop out and major switching decisions after enrollment. There is no previous study that investigates the effects of significant others on non-completion decisions after the initial choice of a major. Third, I will also investigate where students in gender atypical majors go when switching their initially chosen major. To my knowledge, there is no empirical study which investigates a combination of these different aspects.

\section{State of Research}

Evidence on the effect of the gender composition of a major on persistence in that major points to contradictory results. I only present results of studies that either directly measure the effect of sex ratio in a major on persistence, or conduct within-gender comparisons. Some studies found that a low proportion of female students is associated with women experiencing thoughts of quitting and non-completion (Spangler et al., 1978; Meyer et al., 2019). In contrast, other studies demonstrated that women in male dominated majors are more likely to persist than female students in more gender-balanced majors (Rogers et al., 1991), or found no effect (Riegle-Crumb et al., 2016; Sax, 1996). Results for men in gender atypical majors are also mixed. Some evidence points out that men in female dominated majors are more likely to switch majors than their male peers in male dominated fields, and that when they switch, they do so to more gender-balanced majors (Riegle-Crumb et al., 2016). In contrast, another study found that a higher proportion of women in the major promotes men's persistence (Sax, 1996). 
Overall, these previous studies have some limitations. First, some studies used very small, nonrepresentative samples (Rogers et al., 1991; Spangler et al., 1978). Secondly, the studies differ in their operationalization of the effects of tokenism and in the included control variables, which impedes a comparison of the results. Third, the studies use either cross-sectional data or do not make use of the longitudinal structure of the data. Forth, many studies do not measure the exact sex ratio in the major, and instead use the national gender composition in majors or the average gender composition in the majors in their sample as a proxy. I will try to overcome these shortcomings by using longitudinal data and conducting time survival analysis. I will link my data basis with administrative data which provides the exact sex ratio at the time of enrollment in every major in every university in Germany. Additionally, the data basis allows me to control for many other covariates that might bias the observed effect of sex ratio on students' persistence.

Previous multivariate studies on the social integration of students in gender atypical majors have showed mixed results. Some studies found empirical evidence for lower levels of social integration among female students in male dominated majors. Female students in predominantly male majors earn lower grades, are less active in class and spend less leisure time with fellow students than women in more gender-balanced majors (Spangler et al., 1978; Rogers et al., 1991). In contrast, other empirical studies found no effect of the proportion of women in a major on social integration, social self-concept, satisfaction and academic performance for female and male students when controlling for other student characteristics (Rogers et al., 1991; Sax, 1996). Research on the impact of social integration on the attrition of students in gender atypical fields of studies is still scarce. One previous study indicates that social integration cannot explain the higher non-completion rate of students in gender atypical majors (Meyer et al., 2019). However, this study measures social integration only retrospectively and hence the measurement might be biased. I will try to address these shortcomings by using a data set which continuously observers students' social integration over the course of their studies.

To my knowledge, the influence of significant others on students' non-completion decisions in gender atypical majors has not yet been investigated. More generally, empirical evidence suggests that parents and peers affect the educational and occupational aspirations of youth (Sewell et al., 1970; Morgan, 1998; Bozick et al., 2010). Studies have shown that students adjust their STEM-preferences in high school to those of their friends (Raabe et al., 2019). 
Furthermore, encouragement by parents and peers increases the chance of students choosing a STEM high school curriculum or STEM field of study (Gabay-Egozi et al., 2015; Riegle-Crumb et al., 2006; van der Vleuten et al., 2018). Additionally, support from significant others for choosing a STEM field is affected by traditional gender norms. Girls receive less encouragement from parents and peers to choose a STEM major than boys (Gabay-Egozi et al., 2015).

In the next chapter, I will first build a synthesis of Tinto's model of student drop out and Kanter's theory of tokenism. Second, I will describe the theoretical background for the influence of significant others.

\section{Theoretical Background}

\section{A synthesis of Tinto's model of drop out from higher education and Tokenism}

Tinto (1975) argues that low social integration is one of the major determinants of student drop out from higher education. He argues that student drop out is the consequence of insufficient social interaction with fellow students and faculties, as well as insufficient congruency with the values of the educational institution. Tinto calls these two aspects social integration and academic integration. Successful social integration is characterized by positive communication and friendship with fellow students, support from faculties and affiliation to the college collectivity. This paper only focusses on social integration with fellow students. In Germany, students usually do not have contact with faculties outside of class and hence, I expect that faculties are less important for students to feel socially integrated than in the USA - the context in which Tinto developed his theory. Academic integration can be measured by grade performance and intellectual development (Tinto, 1975).

According to Tinto, drop out is a longitudinal process of interaction between the student and the social and academic systems of an educational institution. Students' experience in college, i.e. their social and academic integration, continuously shapes their commitment to the completion of higher education and commitment to a specific educational institution, leading to persistence in the educational institution, or drop out. Social integration and academic integration are often closely interrelated, but they exert an independent influence on drop out decisions (Tinto, 1975). Many empirical studies support Tinto's arguments and show that 
social and academic integration predict students' persistence in higher education (e.g., Chen, 2012; Meyer et al., 2019).

Following Kanter's theory of Tokenism (1977b), I argue that male and female students in gender atypical majors are more likely to experience low social integration with fellow students and hence, have a higher non-completion risk than students in gender typical programs. According to Kanter (1977b), men and women who are in the numerical minority in an environment become "tokens", symbols or representatives of their category. Tokens are seen by the dominant group members as "stand-ins" for their category rather than individuals. Kanter (1977a) observed in an empirical study that Tokenism led to three perceptual tendencies that negatively affected the tokens' performance and career advancement: heightened visibility, polarization and assimilation.

Firstly, tokens attract a certain amount of attention because of their rarity. This increased visibility creates performance pressure for the tokens, with their individual performance being treated as representative of how well all members of the token's category would perform a specific task. This burden can negatively affect tokens' performance levels or conversely, lead to overachievement (Kanter, 1977b). The second perceptual tendency is polarization. The dominant group feels threatened by the token culture. The dominants thus exaggerate the differences between the dominant group and the tokens and heighten the boundaries between them. As a consequence, tokens also face informal isolation. They are excluded from informal socialization occasions and networks by the dominants who want to preserve their superior status (Kanter, 1977b). Third, the dominant group has a perceptual tendency towards assimilation. The tokens' characteristics are distorted to fit the pre-existing stereotypical attributes of their category. This leads to the role encapsulation of tokens: tokens are forced into playing stereotypical roles, such as for female tokens, the mother, the seductress, the iron lady, etc. (Kanter, 1977b).

As this overview demonstrates, tokens face various difficulties in their interaction with dominants. These difficulties are also expected to affect the tokens' feeling of social integration. Tokens might be intentionally socially isolated by dominants who are seeking to protect their culture. Additionally, tokens' high visibility, combined with negative stereotyping, and the emphasis placed on their different and subordinate status in comparison to dominants makes tokens outsiders in a group. They are not respected as 
individuals and their achievements are overlooked. As a consequence, tokens feel pressured to leave the group (Kanter, 1977b).

Kanter only investigated tokens in an occupational environment. In my paper, I apply Kanter's theory of tokenism to undergraduate students, as have previous studies (Rogers et al., 1991; Sax, 1996). Students in gender-atypical fields of study are in the numerical minority. Furthermore, fields of study are associated with gender stereotypes as well as occupations (Correll, 2001). Following Kanter's theory, students in gender-atypical majors are also likely to experience the process of tokenism and face difficulties in their interactions with fellow students of the other gender.

Kanter (1977a) argues that the process of tokenism not only applies to women in male dominated occupations, but also to men in female dominated fields. In contrast, newer research (Williams, 1995) shows that the experiences of male tokens differ from those of female tokens. Male tokens are also confronted with stereotypes, but instead of being pressured to leave the occupation, male tokens are pushed "upward" into managerial positions, to which men are assumed to be more suited (Williams, 1995). This phenomenon is called the "glass elevator". Since undergraduate students generally have equal status, there is no possibility for male tokens to be pushed "upward" into a higher position. Consequently, I follow Kanter and assume that women and men in gender-atypical fields of study experience lower social integration than those in gender-typical or gender-neutral fields of study.

In line with Tinto, (1975), I further hypothesize that low social integration with fellow students increases the non-completion risk of students. If low social integration is caused by the minority status of students in gender atypical majors, not only should drop out from higher education, but also switching to a major with a higher proportion of same sex students solve their problem of low social integration. According to Kanter (1977b), the negative effects of tokenism are less exaggerated when the proportion of tokens in a group increases. I therefore expect that when switching majors, token students are more attracted to gender balanced majors or majors dominated by their own gender than to majors which are equally or more dominated by the other gender. Based on these arguments I derive the following hypotheses: H1a) Women in male dominated majors have a higher risk of dropping out or switching to a major with a higher proportion of women than women in female dominated majors.

H1b) Men in female dominated majors have a higher risk of dropping out or switching to a major with a higher proportion of men than men in male dominated majors. 
H2) Differences in social integration contribute to the explanation of the higher noncompletion risk of students in gender atypical majors.

The impact of significant others on students' non-completion decisions

The influential "Wisconsin Model" (Sewell et al., 1969), as well as newer revisions of the model (Morgan, 1998), emphasize the influence of significant others (henceforth, SOI) on students' educational and occupational status attainment. Significant others are defined as persons that exert great influence on a student, such as parents, teachers, friends or other peers. Parents are still relevant for teenagers and young adults but over time peers gain more and more in importance (Furman et al., 1992). Significant others can act as role models or can directly encourage students to choose a specific educational or occupational path (Woelfel et al., 1971). The Wisconsin model is supported by empirical evidence (Bozick et al., 2010).

Other theories argue that significant others act as comparative and normative reference groups. Comparative reference groups provide a frame of reference which allows an individual to judge problematic issues or the adequacy of one's behavior (Merton et al., 1968). Members of the comparative reference group act as role models, and comparing themselves with this group can lead to individuals altering their behavior to conform with that of members of the reference group (Kemper, 1968). Normative reference groups provide norms and values with which an individual is expected to comply. Deviance from the group norms is sanctioned, for example by social isolation. Through this negative reinforcement, normative reference groups drive conformity to appropriate roles, including appropriate sex roles (Kemper, 1968). Several studies show that parents and peers punish sex-inappropriate behavior (Langlois et al., 1980; Massad, 1981). Children learn gender roles during the process of socialization which are shaped by significant others. Although girls also can achieve well in mathematics and science, gender stereotypes concerning the inferior science skills of women remain. In contrast, men are believed to be less suited for tasks involving caring and communication (Correll, 2001). Consequently, the influence of significant others is biased by traditional gender norms. This is also supported by empirical evidence (Gabay-Egozi et al., 2015). Students in gender atypical majors are acting against prevalent traditional gender norms. Consequently, their choice of major is more likely to meet the disapproval of significant others. Furthermore, they might lack role models who are also following a gender atypical career path. The pressure from parents and peers to comply with traditional gender norms may lead these students to revoke 
their choice of major and to switch to a more gender typical major, or to drop out from higher education. This leads to a third hypothesis:

H3) Pressure from significant others contributes to the explanation of the higher noncompletion risk of students in gender atypical majors.

\section{Data and methods}

I use data from the university student cohort of the German National Educational Panel Study (NEPS-SC5) to test my hypotheses. NEPS-SC5 is a representative panel survey in Germany which follows 17,910 first-year undergraduate students who enrolled in a full university or university of applied science ${ }^{2}$ in the winter term 2010/11. Students are interviewed twice a year. The observation period consists of 6 years. In this time span most students have graduated.

For my analysis, I exclude students who are enrolled in distance studies. Students in this type of institution only have little contact with other students in their major. Hence, social integration should play a negligible role in the persistence decisions of these students. Furthermore, I exclude students who do not report their major or are enrolled in more than one major. In the latter case, it is unclear which of the majors and its corresponding sex ratio causes the observed non-completion. Missing values are imputed for a subset of variables with multiple imputation by chained equations $(m=100)^{3}$. These restrictions and listwise deletion for non-imputed variables leave a sample size of 11,482 students (61\% female and $39 \%$ male).

To estimate the effect of gender composition in a major, and social integration on drop out and switching behavior, I conduct survival analyses with competing risks. This method allows right-censored observations and time-varying covariates to be included in the estimation of non-completion risk. Due to the large amount of ties (events take mostly place at the end of a semester), I apply discrete time survival analysis with 10 time intervals. The time intervals consist of 6 months, except the last one, which pools 18 months. Events rarely occur in the last year when students are already close to graduation. I differentiate three competing risks: 1. Switching to a major with a lower or same proportion of same sex students, 2 . Switching to a major with at least a $5 \%$ higher proportion of same sex students ${ }^{4}$, and 3 . Dropping out from higher education. If students drop out from higher education but enroll again within the observation period, the interruption is ignored and not counted as a drop out. For women, I 
observe 917 events; for men 762. Table 1 provides an overview of the different types of events observed in the data.

Table 1: Distribution of observed events

\begin{tabular}{|c|c|c|c|c|}
\hline & $\begin{array}{c}\text { Event type 1: } \\
\text { Switching to a major with a } \\
\text { lower or same prop. of } \\
\text { same sex students }\end{array}$ & $\begin{array}{c}\text { Event type } 2 \text { : } \\
\text { Switching to a major with at } \\
\text { least } 5 \% \text { higher prop. of } \\
\text { same sex students }\end{array}$ & $\begin{array}{c}\text { Event type } 3: \\
\text { Drop out }\end{array}$ & Total \\
\hline Women & $37,2 \%$ & $32,4 \%$ & $30,4 \%$ & $100 \%$ \\
\hline Men & $39,5 \%$ & $26,1 \%$ & $34,4 \%$ & $100 \%$ \\
\hline
\end{tabular}

Source: doi:10.5157/NEPS:SC5:11.0.0, own calculations

I conduct multinomial logit models with time intervals as covariates. To take the nesting of time intervals in persons into account, I estimate clustered standard errors. I also conducted models with person-specific random intercepts to see if unobserved heterogeneity between clusters and within-cluster dependence biases the results. There seems to be no substantial amount of frailty, and the results do not differ from the analyses with clustered standard errors. Furthermore, the nesting of the individuals in universities only explains around $2 \%$ of the variance. Therefore, I do not include person- or university-specific random intercepts in the models. The analyses are conducted separately for men and women to allow for withingender comparisons.

\section{Covariates}

\section{Gender composition of majors}

I define majors with $30 \%$ or fewer women as male dominated, majors with $70 \%$ or more women as female dominated and majors with more than $30 \%$, but fewer than $70 \%$ women as gender balanced (Riegle-Crumb et al., 2016). If a field is dominated by one gender by $70 \%$ or more, then the general public strongly identifies the field with that gender and corresponding gender stereotypic images (Cejka et al., 1999). To capture the sex ratio in a major, and consequently the conditions of students in a major as precisely as possible, I linked the NEPSSC5 data with external data from the German Federal Statistics Office. The German Federal Statistics Office provides information on the exact sex ratio in each major for each German university in 2010 . For $17 \%$ of the cases, the specific sex ratio in a major is not available. For these cases I used the average sex ratio in a major over all German universities. Following my definition, male dominated majors include engineering programs, physics, informatics and 
technology. Female dominated majors include language programs, pedagogy, psychology, health studies, social sciences and art. Table 2 displays the distribution of women and men over the different types of majors in the final analysis sample. 463 women and 439 men are enrolled in a gender atypical major.

Table 2: Distribution of women and men over majors

\begin{tabular}{lcccc}
\hline Sex ratio major & Women N & Women $\%$ & Men N & Men \% \\
\hline Female dom. & 2,837 & 40 & 439 & 10 \\
Gender balanced & 3,725 & 53 & 2,092 & 47 \\
Male dom. & 463 & 7 & 1,926 & 43 \\
\hline Total & 7,025 & 100 & 4,457 & 100 \\
\hline
\end{tabular}

Source: doi:10.5157/NEPS:SC5:11.0.0, own calculations

\section{Social integration and SOI}

Social integration measures follow Tinto's theoretical model and are adapted for the German context. Three items capture the quantity and quality of interactions with fellow students: "I am able to establish contacts with students", "I speak with classmates about questions in my field of studies", "I have a lot of contact with students" (scales from 1-does not apply at all, to 4 applies completely). I build an index consisting of the mean over all items. Information on social integration is collected once per year.

The influence of significant others is measured as the approval of the choice of major by parents and by friends in the first semester. Students report how much they agree to the statement "my parents/my friends think that I chose a good major" (scales from 1-does not apply at all, to 5 applies completely). I distinguish between negative, medium and positive (reference category) judgement of the major.

\section{Control variables}

I control for background characteristics of the students in all stepwise models: migration background (yes/no), parental education (1: both parents have no tertiary degree, 0 : at least one parent has tertiary degree) and age at enrollment as a centered variable.

Tinto, (1975) argues that not only social, but also academic integration has an impact on noncompletion. Therefore, I include measures of academic integration in the analyses. Academic integration is measured once per year and divided into three different aspects, which are 
included as separate index variables: affective involvement, achievement orientation and expectation fulfillment. Affective involvement uses three items to measure how much students identify with their studies and whether they enjoy studying. Achievement orientation uses three items to capture how much energy students invest in their studies (scales from 1does not apply at all, to 5 applies completely). Expectation fulfilment reflects students' satisfaction with their academic performance. It is measured using three items (scales from 1does not apply at all, to 4 applies completely). For each aspect of academic integration, I build indices with the mean over all items of the construct.

I further control for characteristics of the program students are enrolled in and the university. I consider if students are aiming for a teaching degree (yes/no) and if they are enrolled in a university of applied science or a full university (1: university of applied science, 0 : full university).

Additionally, I control for whether students are working (yes/no) and their self-rated health (very good/average or bad vs. good) because both reflect how much time and energy they have to build social contacts. The employment history of the students is followed up on continuously, health is measured every second wave. Furthermore, students who are studying close to their hometown may have less interest in building contacts within the university because they can maintain frequent contact with their friends in their hometown. I use the district ("Kreise") of the last attended high school as a proxy for the students' hometown and control for the driving distance in minutes between the hometown and the university district where students enrolled in 2010.

Another important factor for self-selection and persistence in higher education is the level of preparation for higher education. Hence, I control for students' high school leaving certificate GPAs, and whether they attended an advanced STEM course during high school finals (no advanced STEM course /no information ${ }^{5}$ vs. advanced STEM course). I only consider hard science as STEM, i.e. mathematics, physics, informatics, technology and general natural science. Furthermore, I take into account whether students completed vocational educational training before enrollment (yes/no).

Additionally, switching decisions might be caused by a mismatch between vocational preferences and the major. I use the RIASEC-scores which are measured in the first wave to assess students' vocational interests. The RIASEC-scores differentiate between six distinct 
vocational interests: realistic, investigative, artistic, social, entrepreneurial and conventional (Holland, 1997). For each vocational interest dimension I build an overall mean score.

\section{Results}

\section{Descriptive Results}

Figure 1 and 2 show the Kaplan-Meier failure estimator separately for female and male students. The figures depict the development of the different attrition rates for students from the first major they enrolled in over the course of the 10 time intervals measured (corresponding to 6 years). For a better illustration, the graphs only contain students in gender typical and atypical majors. Here, I do not yet distinguish between different events. Failure is defined as non-completion in the form of switching to any other major before graduation or dropping out. Overall, the attrition rates for men and women are relatively low at around $25 \%$. Non-completion rates are highest at the beginning of the observation period. Most students decide to leave their initial chosen major in the first 2 years.

In line with my hypotheses, the results for women illustrate that a slightly higher proportion of women in male dominated majors do not complete their major in comparison to women in female dominated majors (figure 1). In contrast, results for men demonstrate that the proportion of men in female dominated majors who do not complete their major is slightly, but not significantly lower than the proportion of men in male dominated majors who experience non-completion (figure 2). In contrast to my expectations, it seems that men in gender atypical majors do not differ from men in gender typical majors in terms of attrition rate. However, these results do not yet reveal the potentially different attrition rates for the three different paths I am interested in after non-completion. 
Figure 1: Attrition of female undergraduate students

Kaplan-Meier survival estimates:

Percentage of female students remaining in the major

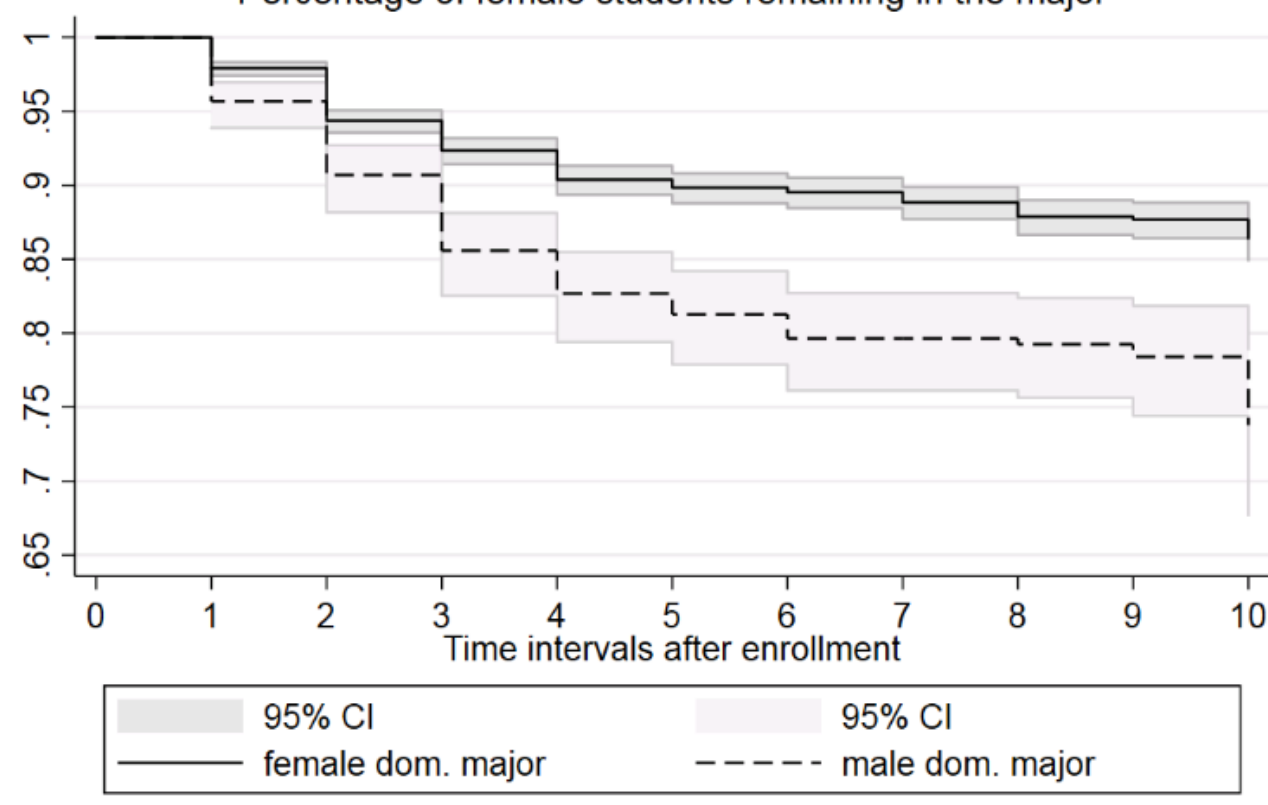

Source: doi:10.5157/NEPS:SC5:11.0.0, own calculations

Figure 2: Attrition of male undergraduate students

Kaplan-Meier survival estimates:

Percentage of male students remaining in the major

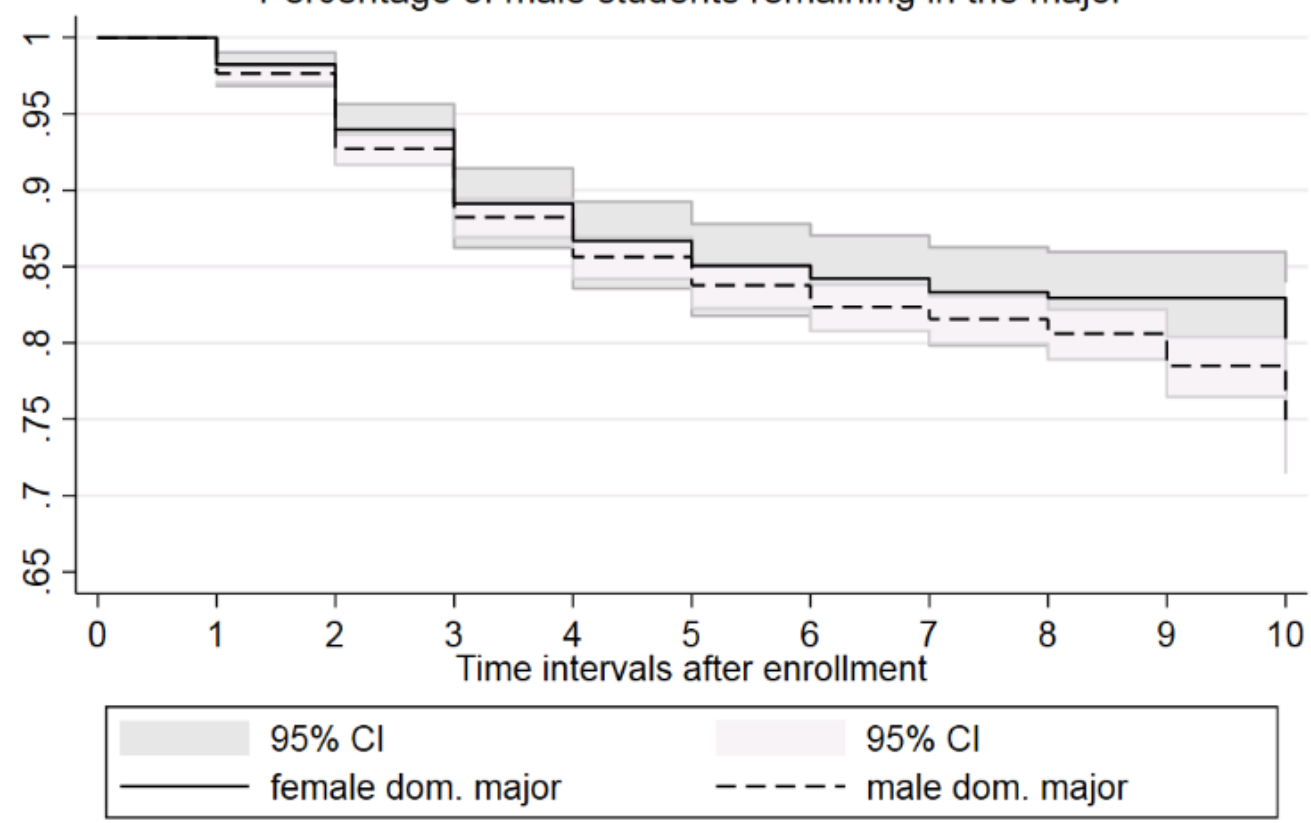

Source: doi:10.5157/NEPS:SC5:11.0.0, own calculations 


\section{Multivariate Results for Female Students}

Table 3 depicts the discrete-time survival analysis with competing risks for female students. Results show average marginal effects, and covariates are introduced stepwise. This allows changes in coefficients when additional controls are included to be observed. Model 1 shows the effect of sex ratio in a major on the different non-completion risks. Women in male dominated majors have a significantly lower risk of switching to a major with the same or lower proportion of women than women in female dominated majors. In line with my hypothesis, women in male dominated majors have a significantly higher risk of switching to a major with an at least $5 \%$ higher proportion of female students, and of dropping out than women in female dominated majors. Non-completion decisions for women in genderbalanced majors show the same pattern. In model 2, social integration with fellow students is included. Good social integration with fellow students significantly decreases the risk of experiencing any of the three events, supporting Tinto's theoretical assumption. However, effect sizes for the sex ratio in the major do not change substantially and remain significant when social integration is controlled for. Consequently, the higher risk of women in male dominated majors of switching to a more gender balanced or female dominated major is not due to lower student social integration levels.

In model 3, SOI, in- and pre-college experience and psychological characteristics are included in the model. The results demonstrate that a low or medium approval of the major by parents and friends increases the risk of switching to a more male dominated major, but also to a major with $5 \%$ more female students. SOI does not predict drop out. Although SOI has an impact on switching decisions, the effect of the sex ratio does not change when SOI is controlled for. The risk of students in male dominated majors of dropping out is reduced in model 3 and is no longer significant. However, this change is caused by other covariates included in model 3, particularly vocational interests. Hence, SOI also cannot explain women in gender atypical majors' higher risk of switching to a more female dominated major or of dropping out. Controlling for academic integration, type of program and university, employment status, health, college preparation and vocational training, and vocational interests does not change the results for sex ratio in the major on switching decisions. On the contrary, the effect becomes even stronger. Female students in gender atypical majors seem to have some characteristics that protect them from an even higher risk of switching to a more gender balanced or female dominated major. 
To sum up, the results partially support hypothesis $1 \mathrm{a}$. Women in gender atypical majors have a significantly higher risk of switching to a major with an at least $5 \%$ higher proportion of women and a higher drop out risk. Hypothesis 2 and 3 must be rejected for women. The higher risk of switching to a more gender balanced major or of dropping out is not caused by lower levels of integration or support from significant others for women in male dominated majors. While the higher drop out risk of women in male dominated majors can be explained by psychological characteristics, other in-college experiences, differences in college preparation and psychological characteristics fail to explain the observed effect of the sex ratio on switching decisions.

\section{Multivariate Results for Male Students}

Table 3 depicts the results of discrete-time survival analysis with competing risks for male students. The results show a similar picture to the analysis for women. Model 1 illustrates that men in female dominated majors have a significantly lower risk of switching to a major with the same or higher proportion of women than men in gender typical majors. In contrast, they have a significantly higher risk of switching to a major with an at least $5 \%$ lower proportion of women than men in male dominated majors. This result is in line with hypothesis $1 \mathrm{~b}$. However, in opposition to my expectations, they do not have a higher drop out risk. The smaller risk of switching to an even more female dominated major explains why descriptive results previously did not show a significant difference in terms non-completion risk between men in female dominated and men in male dominated majors. This demonstrates that is important to look at the different destinations of non-completers instead of only the occurrence of noncompletion. Results for men in gender balanced majors show the same pattern, but only the effect on the risk of switching to a more gender-balanced or male dominated major is significant. Results do not change substantially if social integration is controlled for, as in model 2. Good social integration with others students decreases the risk of experiencing any of the three non-completion events. However, lower integration does not explain the higher risk of males in gender atypical majors switching to more gender balanced or gender typical majors. In conclusion, hypothesis 2 must be also rejected for men. In model 3, SOI, in- and pre-college experiences and psychological characteristics are included. The results illustrate that low and medium levels of approval of the major by friends and parents increases the risk of switching to a more male dominated major, as well as to a more female dominated major. 
However, only the effects for friends are significant. Medium disapproval of the major by parents also increases the drop out risk. In contrast, disapproval of the major by friends seems to decrease the drop out risk. Overall, significant others have an impact on non-completion decisions. Nevertheless, SOI cannot explain the effect of the sex ratio, which remains significant in model 3. In-college experience, employment status, health, college preparation, vocational training, and vocational interests also do not explain the observed effects.

In summary, the empirical evidence partially supports hypothesis 1b. Men in gender atypical majors have a significantly higher risk of switching to a major with an at least $5 \%$ lower proportion of women, but not a significantly higher drop out risk. The results do not support hypothesis 2 and 3 for men. Low social integration and disapproval by significant others do not seem to be the cause for the higher risk of switching to a major with a lower proportion of women for men in female dominated majors. Other in-college experiences, differences in college preparation and psychological characteristics also do not contribute to the explanation of the observed effects.

Overall, the results demonstrate that the assumption of Tokenism, Tinto's model of student drop out and SOI are independently valid. However, neither social integration nor SOI can explain the observed effect of the sex ratio in a major.

The results are robust when I increase the threshold for switching to a major with a higher proportion of same sex students to $10 \%$ instead of $5 \%$ as dependent variables, or when I use the metric sex ratio as an independent variable. I conducted mean comparisons and simple regression models to further explore the puzzling results.

These descriptive analyses demonstrate that women in gender atypical majors are slightly, but significantly better integrated than women in gender typical and gender-balanced majors. Apparently, their minority status does not negatively affect social contacts. Additionally, women in male dominated majors are even significantly less likely to meet the disapproval of parents with their major choice. However there is no difference in the judgement of the major by friends between women in male and female dominated majors. Mean comparisons for male students show a different pattern. Men in female dominated majors do not differ from men in male dominated and gender balanced majors in terms of social integration. Men in gender atypical majors are significantly more likely to meet the disapproval of parents and friends with their choice of major. Apparently, men are more likely to be punished by 
significant others for violating gender norms than women. Nevertheless, this difference does not contribute to the explanation of the sex ratio effect in the multivariate model.

Table 3: Women: discrete-time survival analysis with competing risks

\begin{tabular}{|c|c|c|c|c|c|c|c|c|c|}
\hline & \multicolumn{3}{|c|}{$\begin{array}{l}\text { Event 1: Switching to major } \\
\text { with lower or same prop. of } \\
\text { women }\end{array}$} & \multicolumn{3}{|c|}{$\begin{array}{c}\text { Event 2: Switching to major } \\
\text { with at least } 5 \% \text { higher } \\
\text { prop. of women }\end{array}$} & \multicolumn{3}{|c|}{ Event 3: Drop out } \\
\hline & M1 & $\mathrm{M} 2$ & M3 & M1 & $\mathrm{M} 2$ & M3 & M1 & $\mathrm{M} 2$ & M3 \\
\hline \multicolumn{10}{|l|}{$\begin{array}{l}\text { Sex ratio in major } \\
\text { (Ref.: Female dom.) }\end{array}$} \\
\hline Gender-balanced & $\begin{array}{c}-0.004 * * * \\
(0.001)\end{array}$ & $\begin{array}{c}-0.004 * * * \\
(0.001)\end{array}$ & $\begin{array}{c}-0.005 * * * \\
(0.001)\end{array}$ & {$\left[\begin{array}{c}0.008^{* * *} \\
(0.001)\end{array}\right.$} & $\begin{array}{c}0.008 * * * \\
(0.001)\end{array}$ & $\begin{array}{c}0.009 * * * \\
(0.001)\end{array}$ & $\begin{array}{l}0.002^{*} \\
(0.001)\end{array}$ & $\begin{array}{l}0.002^{*} \\
(0.001)\end{array}$ & $\begin{array}{c}0.001 \\
(0.001)\end{array}$ \\
\hline Male dominated & $\begin{array}{c}-0.006 * * * \\
(0.002)\end{array}$ & $\begin{array}{c}-0.006 * * * \\
(0.002)\end{array}$ & $\begin{array}{c}-0.007 * * * \\
(0.002)\end{array}$ & $\begin{array}{c}0.016^{* * *} \\
(0.003)\end{array}$ & $\begin{array}{c}0.016 * * * \\
(0.003)\end{array}$ & $\begin{array}{c}0.030 * * * \\
(0.006)\end{array}$ & $\begin{array}{l}0.007^{*} \\
(0.002)\end{array}$ & $\begin{array}{l}0.007^{*} \\
(0.002)\end{array}$ & $\begin{array}{c}0.001 \\
(0.002)\end{array}$ \\
\hline Social integration ${ }^{1}$ & & $\begin{array}{l}-0.002^{*} \\
(0.001)\end{array}$ & $\begin{array}{l}-0.002 \\
(0.001)\end{array}$ & & $\begin{array}{c}-0.004 * * * \\
(0.001)\end{array}$ & $\begin{array}{c}-0.004 * * * \\
(0.001)\end{array}$ & & $\begin{array}{c}-0.008 * * * \\
(0.001)\end{array}$ & $\begin{array}{c}-0.005^{* * *} \\
(0.001)\end{array}$ \\
\hline \multicolumn{10}{|c|}{$\begin{array}{l}\text { Approval of the major } \\
\text { by parents (Ref: High) }\end{array}$} \\
\hline Medium & & & $\begin{array}{c}0.003 \\
(0.001)\end{array}$ & & & $\begin{array}{l}0.004^{*} \\
(0.001)\end{array}$ & & & $\begin{array}{c}0.000 \\
(0.001)\end{array}$ \\
\hline Low & & & $\begin{array}{l}0.010^{*} \\
(0.003)\end{array}$ & & & $\begin{array}{l}0.010^{*} \\
(0.003)\end{array}$ & & & $\begin{array}{c}0.002 \\
(0.002)\end{array}$ \\
\hline \multicolumn{10}{|l|}{ by friends (Ref: High) } \\
\hline Medium & & & $\begin{array}{c}0.003^{*} \\
(0.001)\end{array}$ & & & $\begin{array}{l}0.002^{*} \\
(0.001)\end{array}$ & & & $\begin{array}{c}0.000 \\
(0.001)\end{array}$ \\
\hline Low & & & $\begin{array}{c}0.009 * * * \\
(0.003) \\
\end{array}$ & & & $\begin{array}{l}0.006 * \\
(0.002) \\
\end{array}$ & & & $\begin{array}{r}0.001 \\
(0.002) \\
\end{array}$ \\
\hline $\begin{array}{l}\text { Time intervals } \\
\text { and background } \\
\text { variables }\end{array}$ & $\mathrm{x}$ & $\mathrm{x}$ & $x$ & $\mathrm{x}$ & $x$ & $x$ & $x$ & $x$ & $x$ \\
\hline $\begin{array}{l}\text { In- and pre-college } \\
\text { experience }\end{array}$ & & & $x$ & & & $x$ & & & $x$ \\
\hline $\begin{array}{l}\text { Psychological } \\
\text { characteristics }\end{array}$ & & & $x$ & & & $x$ & & & $x$ \\
\hline McFadden $\mathrm{R}^{2}$ adj. & 0.054 & 0.073 & 0.122 & 0.054 & 0.073 & 0.122 & 0.054 & 0.073 & 0.122 \\
\hline $\mathrm{N}$ events & & 341 & & & 297 & & & 279 & \\
\hline $\mathrm{N}$ person-intervals & & & & & 39,957 & & & & \\
\hline N persons & & & & & 7,025 & & & & \\
\hline \multicolumn{10}{|c|}{$* p<0.05, * * p<0.001, * * * p<0.001$} \\
\hline \multicolumn{10}{|c|}{ Source: doi:10.5157/NEPS:SC5:11.0.0, own calculations } \\
\hline \multicolumn{10}{|c|}{$\begin{array}{l}\text { In - and pre-college experience: academic integration }{ }^{1} \text {, working }{ }^{1} \text {, health }{ }^{1} \text {, high school GPA, advanced STEM course in high } \\
\text { school, vocational training completed, distance between hometown and university, aiming for teaching degree }{ }^{1} \text {, type of } \\
\text { university }\end{array}$} \\
\hline \multicolumn{10}{|c|}{ Psychological characteristics: RIASEC vocational interests } \\
\hline Note: ${ }^{1}$ time varying co & & & & & & & & & \\
\hline
\end{tabular}


Table 4: Men: discrete-time survival analysis with competing risks

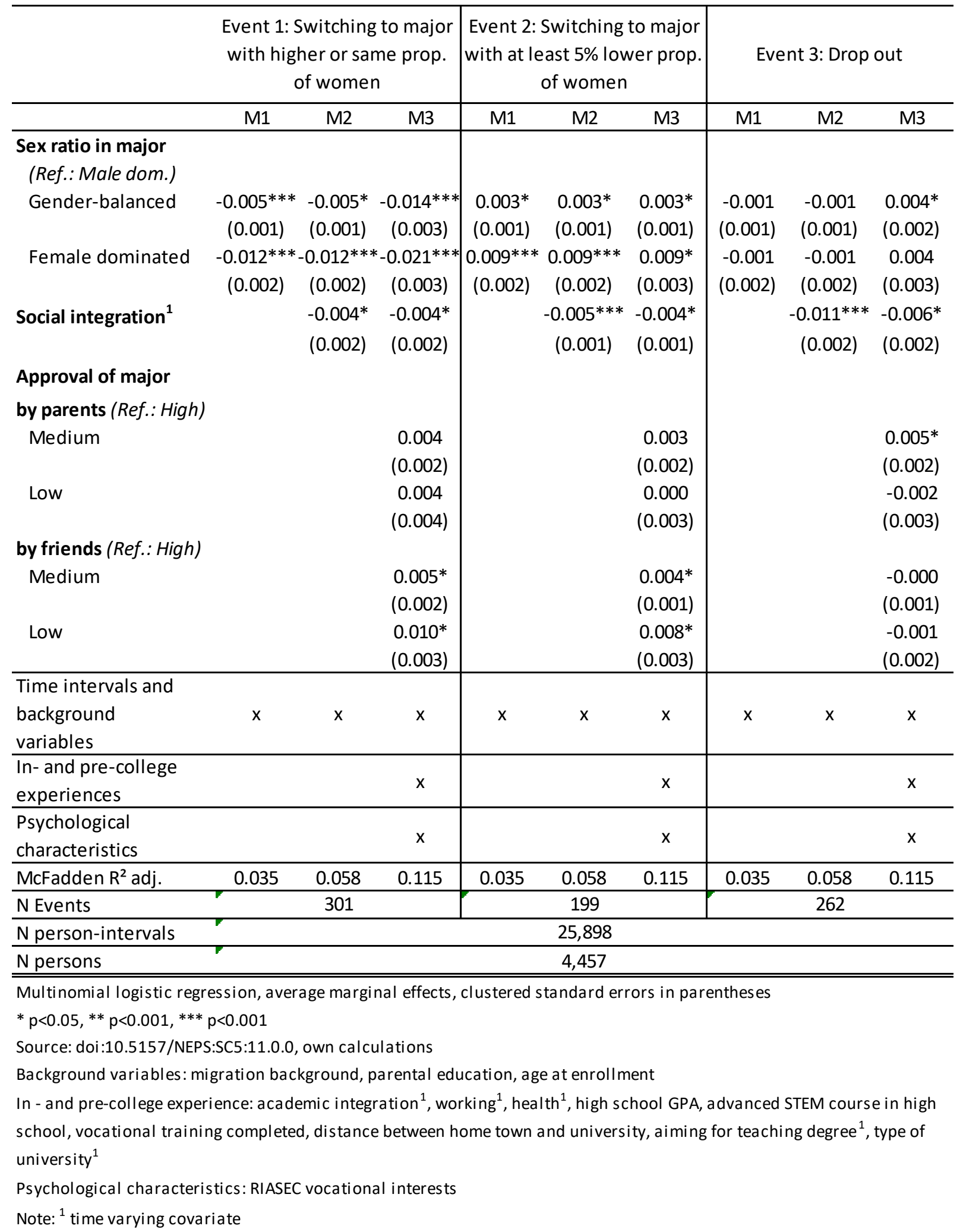




\section{Summary and discussion}

The focus of this paper were students that act against traditional gender norms and enter gender atypical majors. The aim of this study was to find out whether these students are more prone to switching to a major with a higher proportion of students of their gender or to dropping out than students in gender typical majors. Furthermore, I explored whether differences in social integration and SOI might explain the higher non-completion risk of students in gender atypical majors. I conducted within-gender comparisons and estimated discrete-time survival analysis with competing risks to test my hypotheses. In line with my hypothesis, and in contrast to previous studies (Riegle-Crumb et al., 2016), I found that both women and men in gender atypical majors have a significantly higher risk of switching to a major with a higher proportion of students of their gender than students in gender typical majors. Additionally, women in gender atypical majors also have a significantly higher drop out risk. Men in female dominated majors do not have a significantly higher drop out risk than men in male dominated majors. This might be explained by the fact that drop out risks in STEM-fields are in general higher for men and women in comparison to other fields because grades rewarded in these fields tend to be comparatively lower. The results confirm Tinto's model of student drop out and the importance of SOI for students' non-completion decisions. Low social integration with fellow students and disapproval of the major by significant others increases the risk of switching to any major or dropping out. However, lower social integration and SOI do not explain the higher risk of students in gender atypical majors switching to a more gender-balanced or gender typical major. Students in gender atypical majors seem to not experience trouble establishing positive contact with fellow students. Furthermore, students in gender atypical majors, especially women, apparently do not receive substantially less approval for their choice of major from significant others than students in gender typical majors. This might be explained by a selection effect. Students who experience very strong pressure from significant others to comply to traditional gender norms probably will not choose a gender atypical major in the first place.

The causes for the higher risk of students in gender atypical majors switching to a more gender typical or gender balanced major or of dropping out must be caused by factors not observed in my analyses. I will offer two possible alternative explanations for the observed effects.

First, women might decide to leave STEM majors because they realize during the course of their studies that a STEM career is incompatible with their family plans (Ridgeway et al., 2004). 
Men might leave female dominated majors because they become increasingly aware of the smaller economic reward and lesser career opportunities these majors offer in comparison to more gender-balanced majors (Hardie, 2015).

Second, perceived incompatibility between gender identity and the major might cause the higher non-completion risk of students in gender atypical majors. Social identity theory suggests that in an academic setting with a skewed sex ratio, cultural beliefs about gender stereotypes become salient. Affiliation to a gender is seen as relevant for the completion of tasks in that setting. Consequently, people become more aware of societal beliefs about the different capabilities associated with gender (Ridgeway, 2009). Women are believed to have lower skills in science, and men lower care-giving and communication skills (Correll, 2001). Hence, the context communicates an incompatibility between being a women and studying in a STEM field, or being a men and studying pedagogy or language. Previous studies show that a greater perceived identity compatibility with the major predicts intentions to pursue a career in that field (Rosenthal et al., 2013). Unfortunately, my data basis does not provide information on these aspects and I cannot test these assumptions.

Furthermore, my data has some limitations. First, I was only able to observe a small number of events and only few students in a gender atypical major. Second, my measurement of the sex ratio in the majors does not account for the different attrition rates of men and women in a major. When more and more tokens leave gender atypical majors over time, the sex ratio in these majors becomes more and more skewed. Tokens that persist longer in the major might experience greater pressure to leave. However, these tokens might have developed a coping strategy in order to remain (Kanter, 1977a). Hence, I assume that a continuously measured sex ratio might not overly affect the results.

Nevertheless the results do confirm the picture of a "leaky pipeline" for women and men. The few students who chose a pathway contradicting gender norms and enroll in gender atypical studies are more likely to leave this path, opting for a more gender typical field or dropping out. These switching decisions subsequently lead to a further reinforcement of the occupational gender segregation. The explanation for this robust effect should be addressed in further studies with a larger sample of students in gender atypical majors. Future research might focus on exploring other explanations for the higher attrition risk of women and men in gender atypical majors such as gender identity theory. 
${ }^{1}$ This paper uses data from the National Educational Panel Study (NEPS): Starting Cohort Students, doi:10.5157/NEPS:SC5:11.0.0. from 2008 to 2013. NEPS data was collected as part of the Framework Program for the Promotion of Empirical Educational Research funded by the German Federal Ministry of Education and Research (BMBF). As of 2014, NEPS is carried out by the Leibniz Institute for Educational Trajectories (LIfBi) at the University of Bamberg in cooperation with a nationwide network.

${ }^{2}$ Universities of applied science offer a limited number of programs, mostly engineering, management and social work and are more practically oriented.

${ }^{3}$ The following variables are imputed: age at enrollment, social integration, academic integration, high school GPA, vocational interests, distance between hometown and university.

${ }^{4}$ Switching to a major with a higher proportion of same sex students which is still less than $5 \%$ is included in event 1 . Switching university without switching major is not counted as an event.

${ }^{5}$ In some secondary educational institutions, students cannot choose advanced courses in specific subjects for their final exams. For these students information on advanced STEM preparation is not available.

\section{Acknowledgement}

The author wishes to thank Corinna Kleinert for valuable comments and Johannes Hoffmann for the help in calculating the distance measures. 


\section{References}

Bloom, N. (2013). Should I stay or should I go? The role of academic discipline and gender in student attrition and expected earnings, American Sociological Association, Conference Papers, 1-21.

Bozick, R., Alexander, K., Entwisle, D., Dauber, S. and Kerr, K. (2010). Framing the future: revisiting the place of educational expectations in status attainment, Social Forces, 88, 20272052.

Cejka, M. A. and Eagly, A. H. (1999). Gender-stereotypic images of occupations correspond to the sex segregation of employment, Personality and Social Psychology Bulletin, 25, 413423.

Chen, R. (2012). Institutional characteristics and college student dropout risks: A multilevel event history analysis, Research in Higher Education, 53, 487-505.

Correll, S. J. (2001). Gender and the career choice process: The role of biased selfassessments, American Journal of Sociology, 106, 1691-1730.

Furman, W. and Buhrmester, D. (1992). Age and sex differences in perceptions of networks of personal relationships, Child development, 63, 103-115.

Gabay-Egozi, L., Shavit, Y. and Yaish, M. (2015). Gender differences in fields of study: the role of significant others and rational choice motivations, European Sociological Review, 31, 284297.

Hardie, J. H. (2015). Women's work? Predictors of young men's aspirations for entering traditionally female-dominated occupations, Sex Roles, 72, 349-362.

Holland, J. L. (1997). Making vocational choices: A theory of vocational personalities and work environments. New Jersey: Prentice-Hall, INC.

Kanter, R. M. (1977a). Men and women of the corporation. New York: Basic Books.

Kanter, R. M. (1977b). Some effects on proportions on group life: skewed sex ratios and responses to token women, American Journal of Sociology, 82, 965-990.

Kemper, T. D. (1968). Reference groups, socialization and achievement, American Sociological Review, 33, 31-45. 
Langlois, J. H. and Downs, A. C. (1980). Mothers, fathers, and peers as socialization agents of sex-typed play behaviors in young children, Child development, 51, 1237-1247.

Massad, C. M. (1981). Sex role identity and adjustment during adolescence, Child development, 52, 1290-1298.

Merton, R. K. and Rossi, A. S. (1968). Social theory and Social structure. New York: Free Press. Meyer, J. and Strauß, S. (2019). The influence of gender composition in a field of study on students' drop-out of higher education, European Journal of Education, 54, 1-14.

Morgan, S. L. (1998). Adolescent educational expectations: rationalized, fantasized, or both?, Rationality and Society, 10, 131-162.

OECD (2018). Education at a glance 2018. OECD Indicators, OECD Publishing, Paris.

Raabe, I. J., Boda, Z. and Stadtfeld, C. (2019). The social pipeline: how friend influence and peer exposure widen the STEM gender gap, Sociology of Education, 92, 105-123.

Ridgeway, C. L. (2009). Framed before we know it: how gender shapes social relations, Gender \& Society, 23, 145-160.

Ridgeway, C. L. and Correll, S. J. (2004). Unpacking the gender system: a theoretical perspective on gender beliefs and social relations, Gender \& Society, 18, 510-531.

Riegle-Crumb, C., Farkas, G. and Muller, C. (2006). The role of gender and friendship in advanced course taking, Sociology of Education, 79, 206-228.

Riegle-Crumb, C., King, B. and Moore, C. (2016). Do they stay or do they go? The switching decisions of individuals who enter gender atypical college majors, Sex Roles, 74, 436-449.

Rogers, S. J. and Menaghan, E. G. (1991). Women's persistence in undergraduate majors: the effects of gender-disproportionate representation, Gender and Society, 5, 549-564.

Rosenthal, L., Levy, S. R., London, B., Lobel, M. and Bazile, C. (2013). In pursuit of the MD: the impact of role models, identity compatibility, and belonging among undergraduate women, Sex Roles, 68, 464-473.

Sax, L. (1996). The dynamics of "tokenism": how college students are affected by the proportion of women in their major, Research in Higher Education, 37, 389-425. 
Sewell, W. H., Haller, A. O. and Ohlendorf, G. W. (1970). The educational and early occupational status attainment process: Replication and revision, American Sociological Review, 1014-1027.

Sewell, W. H., Haller, A. O. and Portes, A. (1969). The educational and early occupational attainment process, American Sociological Review, 82-92.

Spangler, E., Gordon, M. A. and Pipkin, R. M. (1978). Token women: an empirical test of Kanter's hypothesis, American Journal of Sociology, 84, 160-170.

Tinto, V. (1975). Dropout from higher education: A theoretical synthesis of recent research, Review of Educational Research, 45, 89-125.

van der Vleuten, M., Steinmetz, S. and van de Werfhost, H. (2018). Gender norms and STEM: the importance of friends for stopping leakage from the STEM pipeline, Educational Research and Evaluation, 24, 417-436.

Williams, C. (1995). Still a man's world. Berkeley: University of California Press.

Woelfel, J. and Haller, A. O. (1971). Significant others, the self-reflexive act and the attitude formation process, American Sociological Review, 36, 74-87. 